\title{
Short-term treatment with a beta-block improves cardiac remodeling in arterial hypertension via inhibition of Akt/NF-kB and NFATc4
}

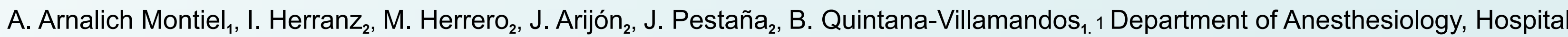
General Universitario Gregorio Marañón, Madrid (Spain), 2 Department of Pharmacology, Medical School of Complutense University, Madrid (Spain)

Background and Goal Study: Our group has previously demonstrated that short-term treatment with esmolol reduces left ventricular hypertrophy (LVH) in spontaneously hypertensive rats (SHRs)(1). However, molecular mechanisms of this effect have not been analyzed to date. The present study is aimed to assess whether short-term treatment with esmolol reverses LVH in aged SHR by down-regulation of Akt/NF-kB and NFATc4 activity.

Materials and Methods: Fourteen-month-old male SHRs were treated intravenously with saline as vehicle (SHR) or esmolol (SHR-E) (300 $\mathrm{mg} / \mathrm{kg} / \mathrm{min})$. Age-matched vehicle-treated male Wistar-Kyoto (WKY) rats served as controls. After 48 hours of treatment, the hearts were harvested and left ventricular tissue separated and processed for Western blot analysis to determine the levels of Akt, NF-kB, NFATc4, Creb1, Serca2a, Erk1/2, Sapk/Jnk. The parameters were compared using single-factor analysis of variance and a post hoc Bonferroni correction was applied. All data were expressed as mean \pm SEM, P< 0.05 was considered significant. All procedures were approved by the Ethics Committee of Hospital General Universitario Gregorio Marañon, Madrid, Spain.

Results and Discussion: Esmolol had the following effects on LVH: a) It reversed the levels of $p-N F A T c 4$ in SHR rats to the control WKY levels, but did not modify the expression of p-Creb1 and Serca2a in SHR rats; b) It also reversed the levels of p-Akt and p-NF-kB in SHR rats to the phospho-levels of these proteins in WKY rats without modifying $\mathrm{p}$-Erk1/2 or $\mathrm{p}$-Sapk/Jnk.

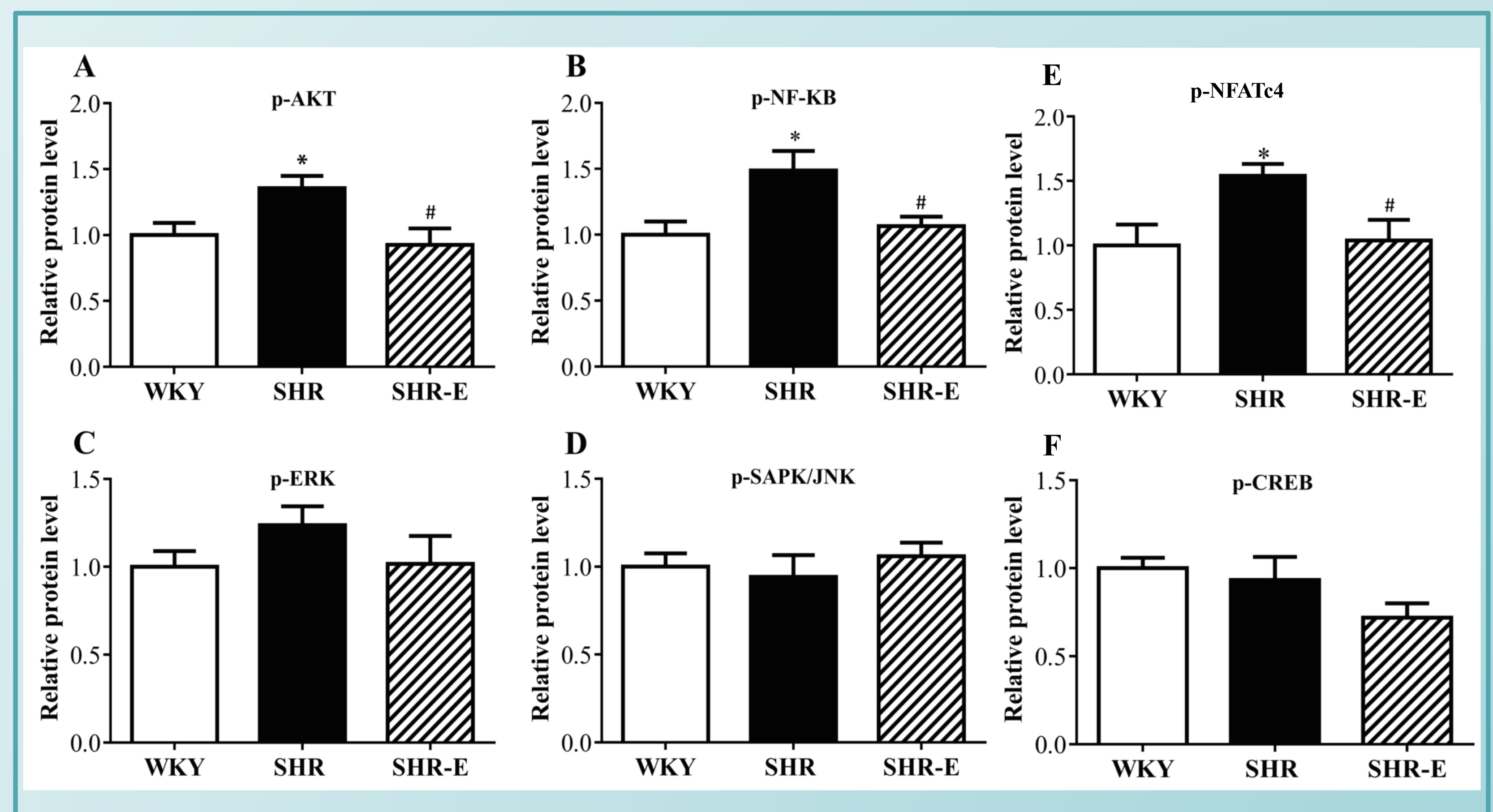

Conclusion: Short-term treatment with esmolol reverses LVH in aged SHR rat by down-regulation of Akt/NF-kB and NFATc4 activity.

Acknowledgements: This work was supported by a grant from PI16/02069 \& Fondos FEDER, Spain.

1. Quintana-Villamandos B, Delgado-Martos MJ, Sánchez-Hernández JJ, Gómez de Diego JJ, Fernández-Criado MdelC, Canillas F, Martos-Rodríguez A, Delgado-Baeza E. Early regression of left ventricular hypertrophy after treatment with esmolol in an experimental rat model of primary hypertension. Hypertens Res Int. 2013; 36(5): 408-13. 